\title{
FAMILY MEDICINE APPROACH BASED ON A WEB-SERIES REVIEW: A CASE REPORT ON HYPEREMESIS GRAVIDARUM
}

\section{Raden Roro Litiya Parahita Putri Firnadi ${ }^{1}$, Nugraeni Affianti ${ }^{1}$, Azimatul Karimah*2, 3}

${ }^{1}$ Faculty of Medicine, Universitas Airlangga, Indonesia, ${ }^{2}$ Coordination Beureau of Community Medicine, Faculty of Medicine, Universitas Airlangga, Indonesia, ${ }^{3}$ Department of Psychiatry, Faculty of Medicine, Universitas Airlangga-Dr. Soetomo General Academic Hospital, Indonesia

\begin{tabular}{|c|c|}
\hline AB & \\
\hline $\begin{array}{l}\text { Family medicine (FM) is a patient-centered and community- } \\
\text { based clinical method. The FM has multiple strengths that are } \\
\text { useful for medical students, such as provides continuing care to } \\
\text { patients and families. Because of the COVID-19, medical } \\
\text { students have been removed entirely from the clinical learning } \\
\text { environment. Online teaching has played key roles in medical } \\
\text { education by covered key clinical conditions, case studies, and } \\
\text { examinations. According to the latest American College of } \\
\text { Obstetricians and Gynecologists (ACOG), Hyperemesis } \\
\text { Gravidarum (HG) is the term for the most severe form of nausea } \\
\text { and vomiting of pregnancy. We used the method to collect data } \\
\text { for a case report taken from a web-series review on Netflix, titled } \\
\text { "Virgin River", Season 2, Episode } 1 \text { to 3. From the symptoms } \\
\text { and physical examination of the patient, Ms. CR was diagnosed } \\
\text { with HG complicated by dehydration, cardiac arrhythmia, and } \\
\text { hypoglycemia. The treatment that has been given to her includes } \\
\text { non-pharmacological and pharmacological therapy. The family } \\
\text { medicine approach has an important role because it has a strong } \\
\text { mutual trust based on the doctor-patient relationship. Also, the } \\
\text { family has a significant role in the treatment of patients since it }\end{array}$ &  \\
\hline
\end{tabular}

\section{INTRODUCTION}

Family medicine (FM) is a patient-centered and community-based clinical method. The FM has multiple strengths that are useful for medical students, such as providing continuing care to patients and families ${ }^{1}$ also addressing physical, psychological, and social problems. ${ }^{2}$ However, because of the COVID-19, medical students have been removed entirely from the clinical learning environment. ${ }^{3}$ This resulted in limited patient care due to the focus on COVID-19 patients and a lack of clinical skills through observation and examination. Working closely with real patients at the hospital is crucial to developing and refining essential skills. ${ }^{4}$ Meanwhile, during the COVID-19, medical students could not directly apply the knowledge they already had. So, online teaching has played a key role in medical education, demonstrated several benefits in enhancing student learning. Teaching sessions have covered key clinical conditions, case studies, and examination questions via live-streamed tutorials through platforms such as Zoom meetings. ${ }^{5}$ 
Online learning is quite challenging, especially for medical students who need hands-on, real-life clinical experience. Meanwhile, this online learning sometimes bored the medical student. "Virgin River" is one of the medical genre web-series that shows the family medicine approach compared to the other web series with the same genre, which focuses more on medical cases in hospitals. This web series also shows the important roles of healthcare providers, families, and the environment in treating the patient.

According to the latest American College of Obstetricians and Gynecologists (ACOG) article on Morning Sickness: Nausea and Vomiting of Pregnancy, HG is the term for the most severe form of nausea and vomiting of pregnancy. $^{6}$ This condition may be diagnosed when a woman has lost $5 \%$ of her pre-pregnancy weight and has other problems related to dehydration or loss of body fluids. ${ }^{6}$ The etiology of HG remains unclear, although hormone changes, changes in the gastrointestinal system, psychological conditions, and genetics are suspected. ${ }^{7}$ In severe cases of $\mathrm{HG}$, maternal complications include vitamin deficiency, dehydration, and malnutrition. For fetal complications of $\mathrm{HG}$, the incidence of low birth weight and premature infants is included. According to the severity of symptoms, $\mathrm{HG}$ can be divided into three levels. The first-line pharmacologic therapy should include a combination of vitamin B6 (pyridoxine) and doxylamine. ${ }^{8}$

\section{MATERIALS AND METHODS}

The author conducted a case report study based on a web-series review as a form of online learning on family medicine modules for medical students. This study has not received ethical clearance from the ethics committee of the Faculty of Medicine,
Universitas Airlangga, because the subjects of this study did not include humans or animals in real life. The data for this study was collected from a web series titled "Virgin River," Season 2, Episode 1 to 3 by two medical students who currently are still doing online learning. This study used the documentary method by watching several episodes while taking important notes about the family medicine approach for HG that appeared in this web series. This web series is available on one of the digital platforms, Netflix. This web series tells the story of Melinda Monroe (MM), a nurse practitioner and midwife who uproots her big-city existence after the death of her husband and chooses to start over by taking a job to assist local family physician doctor Vernon Mullins (VM) in the small, very remote California town of Virgin River. The first to third episodes from season 2 mainly showed how doctors and nurse practitioners gave a homecare treatment for a female patient, Charmaine Roberts (CR), with HG while pregnant with Jack Sheridan (JS) 's baby after they broke up. Her breakup caused depression and tension between her and the nurse, her ex-boyfriend's new love interest.

\section{RESULTS}

The story of this web series focused on the family medicine approach given by the doctor and nurse to a patient with $\mathrm{HG}$ named Ms. CR, unmarried, who came to the clinic with chief complaints of an extreme form of morning sickness caused by nausea and vomiting during her first semester of pregnancy. Although she had been given medication for nausea and vomiting, the complaints did not improve. The other symptoms that she had were migraines, fatigue, loss of appetite, and weight loss. On the first day after receiving homecare treatment, she felt nauseated when lying down, but it was not as bad as before. She 
also could not hold her vomit because it happens continuously. On the second day after she received homecare treatment, the frequency of vomiting was reduced, the patient has only vomited once in the last few hours, but she still felt nauseated when laying down. On that day, she felt fatigued and weak. On the fifth day after she received homecare treatment, the complaints of nausea and vomiting had decreased. However, she complained of heart palpitations.

She looked pale, had low blood pressure, sunken eyes, and acral coldness from the physical examination. Therefore, she was advised to rehydrate by giving intravenous fluids. However, at first, she refused it because she had an excessive fear of needles. Then the patient was given the option to be taken to the hospital for anesthesia before the infusion was placed. She also had mild cardiac arrhythmia based on her complaint of heart palpitations on the fifth day after receiving homecare treatment.

After that, the doctor performed an Obstetric and Gynecology examination by doing an Ultrasound examination, and the result, she had a twins pregnancy. The doctor also suggested a supporting examination, such as a Complete Blood Count (CBC) and blood sugar level, to find out the cause of the cardiac arrhythmia. After the laboratory results came out, the results of $\mathrm{CBC}$ were normal, but she had slightly low blood sugar. So, the cause of the mild arrhythmia was a symptom due to low blood sugar.

She had been diagnosed with HG complicated by dehydration, cardiac arrhythmia, hypoglycemia through patient history, physical, and supporting examination. Her treatment was pharmacological and non-pharmacological, such as Intravenous continuous fluids (IV) of normal saline to restore hydration, medications such as antiemetic, aromatherapy meditation, and reflexology. The patient was given an option to do an aromatherapy meditation and reflexology to overcome her excessive fear of needles and hospital and make her feel less anxious and panic.

The doctor and nurse are daily monitoring blood sugar, ultrasound scan, and symptoms of dehydration or complications. Then, she was advised to do an HG III diet, drink can also be given with meals, and meals should be small portions and frequent. After she received homecare treatment, her complaints of nausea and vomiting were improved, no complaint of palpitation, and her blood sugar level was normal. So, she has been discharged from home treatment, but she has to do regular check-ups and discuss the labor and delivery plan. A few days after being discharged, she went to the clinic and asked for home labor and delivery, but her partner did not agree with the plan, according to the nurse, it was very likely that the twins would be born prematurely and at least one baby would be in the breech position, and to avoid this possibility, she had to undergo a cesarean section. After she was given education and reassurance, she finally agreed to give birth at the clinic accompanied by the doctor and doula, and an ambulance was also prepared to take her and the babies to the hospital if needed.

\section{DISCUSSION}

In this case, the patient has chief complaints of an extreme form of morning sickness that cause nausea and vomiting during her first semester of pregnancy. Nevertheless, the complaints did not improve, even though she had been given medication for nausea and vomiting. The other symptoms that she had were migraines, fatigue, loss of appetite, and 
weight loss. Her symptoms, especially complaints of an extreme form of nausea and vomiting, lead to HG. The onset of these symptoms typically occurs between 6 and 8 weeks gestation and peaks by 12 weeks. Most women experience relief of symptoms by 20 weeks gestation. However, $10 \%$ to $20 \%$ of affected women experience symptoms throughout pregnancy, and symptoms may even persist postpartum. ${ }^{8}$

The primary goal of $\mathrm{HG}$ treatment is symptomatic relief without adverse fetal or neonatal outcomes. Because the etiology of HG remains unclear, the treatment should include non-pharmacological and pharmacological therapy. Nonpharmacological interventions such as dietary, lifestyle modification, acupressure, acupuncture, and ginger. ${ }^{8}$ According to ACOG, the first-line pharmacologic treatment for $\mathrm{HG}$ should include a combination of vitamin B6 (pyridoxine) and doxylamine. Three dosing regiments are endorsed by ACOG, including pyridoxine 10 to $25 \mathrm{mg}$ orally with $12.5 \mathrm{mg}$ of doxylamine three or four times per day, $10 \mathrm{mg}$ of pyridoxine and $10 \mathrm{mg}$ of doxylamine up to 4 times per day, or $20 \mathrm{mg}$ of pyridoxine and 20 $\mathrm{mg}$ of doxylamine up to 2 times per day. The safety of these common drugs is widely accepted, implying no adverse maternal or fetal impacts, but individual studies have raised safety concerns. And these drugs are listed as one of the few FDA pregnancy category A medications. ${ }^{7}$ The combination of pyridoxine and doxylamine is not available in Indonesia, so the general practitioner/doctor in primary healthcare can give pyridoxine, promethazine, and dimenhydrinate instead of doxylamine. ${ }^{9}$ In the case of $\mathrm{HG}$ with dehydration, intravenous fluid boluses or continuous infusions of normal saline should be given in addition to intravenous antiemetics. ${ }^{10}$ For this case, the patient has already been given non-pharmacological and pharmacological treatment such as continuous intravenous fluids (IV) of normal saline to restore hydration, aromatherapy meditation, reflexology, and medications such as antiemetic.

The culture and tradition, in this case, are based on American culture and tradition (Western culture), where having children without marriage is a common thing, and sexual intercourse is regarded as a fundamental human need to be fulfilled for pleasure. In contrast, in some countries, including Indonesia, sexual activity is forbidden before marriage and is only viewed as a means to reproduction. It is also considered taboo because Indonesia upholds Eastern culture and tradition. Despite that situation, even though the patient is unmarried and pregnant with her exboyfriend's babies, the doctor and nurse still give information to her partner about her condition and the treatment that has been given, they also let her partner get involved in making decisions about the labor and delivery process plan so that the final decision is not only taken unilaterally from the patient or the doctor alone. Based on this case, the healthcare provider already fulfills some principles of family medicine, such as giving compassionate care to the patient and her family expressed as empathy and patientcentered communication, the doctor also gives reflective mindfulness of the patient's thoughts and emotions by listening attentively and act with compassion, technical competence, and insight. The healthcare provider also lets the patient's partners get involved in decision-making that can help patients reduce pain and discomfort, faster recovery in physical health, and improvements in emotional health. ${ }^{11}$

Doctor-patient communication is one of the competencies that doctors must master. Effective communication is expected to 
overcome the obstacles experienced by both parties. In providing medical services, effective communication between doctors and patients is a common condition to manage health problems with patients based on patient needs. ${ }^{12}$ The communication between the healthcare provider and patient, in this case, is good because communication is two-way between doctor and patient. The patient has also been explained the diagnosis and the complication, doctor and nurse also provide several alternative options regarding the treatment to be given. The communication between the healthcare provider and the patient's partner in the film is also good.

Implementing the family medicine approach through online learning using this web-series review method is useful in building a humanistic perspective of doctoring and improving medical students' knowledge about the family medicine approach from the healthcare provider. From this web series, the medical student can get a clear image of how to handle a case of HG using the family medicine approach from diagnosing the patient to giving a prompt treatment not only pharmacologically but also nonpharmacologically that already follow the ACOG recommendation for HG. This web series also implement a patient-centered and community-based clinical method that conforms to the principles of family medicine. So, it can be useful for medical student to improve their knowledge about family medicine. However, this study also has its limitations. Because the genre of this web series is medical drama and romantic drama, the information about the medical cases was not detailed and scattered because the story of this web series is also about internal conflict between each character's love life. So, to fully understand the full case, students had to watch several episodes and spend quite an abundant amount of time.

\section{CONCLUSION}

The case from the "Virgin River" web series shows a good example and clear image of the family medicine approach. The family medicine approach has an important role because it has a strong mutual trust based on the doctor-patient relationship. Also, the family has a significant role in treating patients since it can provide effective psychological and emotional support.

\section{REFERENCES}

1. Hellenberg D, Williams FR, Kubendra $\mathrm{M}$, et al (2018). Strengths and limitations of a family physician. Journal of family medicine and primary care, 7, 284-287

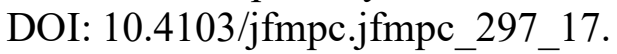

2. World Health Organization. Regional Office for South-East Asia (2003). Family medicine : Report of a Regional Scientific Working Group Meetingon Core Curriculum Colombo, Sri Lanka, 9-13 July 2003. WHO Regional Office for South-East Asia. Available from https://apps.who.int/iris/handle/10665/2 05063.

3. Alsoufi A, Alsuyihili A, Msherghi A, et al (2020). Impact of the COVID-19 pandemic on medical education: Medical students' knowledge, attitudes, and practices regarding electronic learning. PLoS One, 15(11), e0242905 DOI: 10.1371/journal.pone.0242905.

4. Jeyakumar Y, Sharma D, Sirianni G, et al (2020). Limitations in virtual clinical skills education for medical students during COVID-19. Canadian medical education journal, 11(6), e165-e166 DOI: $10.36834 / \mathrm{cmej} .70240$.

5. Dost S, Hossain A, Shehab M, et al (2020). Perceptions of medical students towards online teaching during the COVID-19 pandemic: a national crosssectional survey of 2721 UK medical students. BMJ Open, 10, e 042378 DOI: 10.1136/bmjopen-2020-042378. 
6. Gynecologists ACoOa (2020). Morning Sickness: Nausea and Vomiting of Pregnancy. Available from https://www. acog.org/womens-health/faqs/morningsickness-nausea-and-vomiting-ofpregnancy (Accessed August, 25 2021)

7. Jennings LK, Mahdy H (2021). Hyperemesis Gravidarum. Treasure Island (FL): StatPearls Publishing

8. Castillo MJ, Phillippi JC (2015). Hyperemesis gravidarum: a holistic overview and approach to clinical assessment and management. J Perinat Neonatal Nurs, 29(1), 12-22 DOI: 10.1097/jpn.0000000000000075.

9. Ministry of Health, Republic of Indonesia (2015). Panduan Praktik Klinis Bagi Dokter di Fasilitas Pelayanan Kesehatan Tingkat Pertama, 688-693
10. McParlin C, O’Donnell A, Robson SC, et al (2016). Treatments for Hyperemesis Gravidarum and Nausea and Vomiting in Pregnancy: A Systematic Review. JAMA, 316(13), 1392-1401

DOI:10.1001/jama.2016.14337.

11. Hashim MJ (2016). Principles of family medicine and general practice - defining the five core values of the specialty. J Prim Health Care, 8(4), 283-287

DOI: $10.1071 / \mathrm{hc} 16006$.

12. Fourianalistyawati E (2012). Komunikasi yang Relevan dan Efektif antara Dokter dan Pasien. Psikogenesis, $1,82-87$ 